\title{
A comparative investigation on the effect of thermal treatments on the mechanical properties of oil palm fruitlet components
}

\begin{abstract}
An assessment of the mechanical properties was employed as the principal yardstick to quantify the physical attributes of oil palm fruitlet components before and after being subjected to thermal treatment process. Two industrial-scale thermal treatment methods were considered; horizontal sterilisation (being the most common) and vertical sterilisation (recently implemented). The exocarp and mesocarp layers of thermally-treated fruitlets, as attested by texture profile analysis, compression test, and tensile test, have a significantly low value of fracturability, hardness, firmness, and strength as opposed to untreated fruitlets. Micrographs of the mesocarp slices have provided plausible explanation for the observed decline in the mechanical properties of the sterilised fruitlets. The oil which was initially contained within the fruitlets tends to leach out during the sterilisation process due to the resulting structural disintegration within the layers of the fruitlets. On another related enquiry, the required cracking force to break palm nuts in order to extract the kernel was found to be influenced by the moisture content.
\end{abstract}

Keyword: Oil palm fruitlet; Thermal treatment; Mechanical properties; Structural change; Oil loss 